\title{
Improvement in vernier acuity with practice
}

\author{
SUZANNE P. McKEE and GERALD WESTHEIMER \\ Department of Physiology-Anatomy, University of California, Berkeley, California 94720
}

\begin{abstract}
The ability to detect small differences in the positions of two lines (vernier acuity) showed some improvement with practice in all eight subjects, even for subjects given no error feedback. The average decline in threshold with training $(2,000-2,500$ responses) was about $40 \%$. We used three target orientations: vertical, horizontal, and right oblique. Orientational differences remained stable in only one subject. In five subjects, orientational differences present at the beginning of training diminished or disappeared with increased experience; in two, they increased.
\end{abstract}

In 1863 , Volkmann reported that his resolution acuity and that of his assistant had improved with practice. It was an observation that would be made by many experimenters in subsequent years (Gibson, 1953). Anticipating the signal detection theorists, Wilcox (1936) attributed the change in two-bar acuity he found with practice to a shift in the subject's criterion for "doubleness." Certainly, most of the practice effects observed with the simple psychophysical judgments involved in increment and absolute threshold measurements are indications only of a liberalized standard employed by the subject. If criteron-free measures are used, such thresholds are remarkably stable (Green \& Swets, 1966). On the other hand, practice does indeed benefit sensitivity in tasks which require the identification of a complicated pattern (Gibson, 1969; Tanner \& Rivette, 1963).

Whatever may be responsible for the influence of practice in visual resolution, training effects in hyperacuity could well be of a different origin. The spatial localization of a feature to an accuracy of one-fifth the width of a photoreceptor would seem to demand more complex neural processing than that needed for ordinary acuity. Although we have regularly used a type of forced-choice technique to determine vernier and other hyperacuity thresholds, we have noticed a gradual decrease in the minimum positional difference our subjects can detect, given increased experience with our targets. This paper describes the results of a systematic study of learning in a typical hyperacuity task-vernier offset detection.

In vernier acuity and related hyperacuity judgments, thresholds are usually higher for targets oriented obliquely than for horizontal or vertical targets (Best, 1900; Leibowitz, 1955; Andrews, 1967; Ludvigh \& McKinnon, 1967). We were curious to discover if practice would remove this orientational

This research was supported by the National Eye Institute, U.S. Public Health Service, through Grant EY-00220. difference. Our subjects were thus given training on vertical, horizontal, and oblique targets.

\section{METHODS}

The vernier targets used in these experiments consisted of two bright lines, $19 \mathrm{~min}$ of arc in length, separated by a 1-minof-arc gap and displayed on a dark background. The lines were arranged in one of three orientations: vertical, horizontal, or oblique, i.e., $43^{\circ}$ above the horizontal to the subject's right. Targets were created under computer control on a 602 Tektronix cathode-ray tube equipped with a P4 phosphor. The target lines were actually composed of a row of small dots, each about $1 \mathrm{~min}$ in diameter. These aligned dots were only $30 \mathrm{sec}$ of arc apart and so appeared continuous. The dots could be positioned to an accuracy of $2 \mathrm{sec}$ of arc in the horizontal direction and $1.6 \mathrm{sec}$ vertically, a scale which determined the minimum misalignment of the target lines. The stimulus luminance was about $40 \mathrm{~mL}$ on a background of $0.1 \mathrm{~mL}$. Moderate room illimination was supplied by overhead fluorescent lighting. The luminance of those portions of the apparatus immediately adjacent to the CRT screen was $1 \mathrm{~mL}$. Thus, stability of accommodation was insured by the visible surrounding components of the apparatus. The brevity of the stimulus $(220 \mathrm{msec})$ prevented the initiation of pupillary or oculomotor responses during target presentation.

Subjects sat $2.5 \mathrm{~m}$ from the CRT screen, and viewed the display binocularly with natural pupils. No headholder or bite-bar arrangement was employed during training because we wished the subjects to remain comfortable so they could concentrate on the targets. A check test performed on one observer at the end of training indicated that there was no significant difference between thresholds obtained with or without a headholder for any of the target orientations.

During an experimental run, a target was presented every $3 \mathrm{sec}$ for a duration of $220 \mathrm{msec}$; in the intervening period, there appeared a fixation pattern made of four bright dots which outlined a square $45 \mathrm{~min}$ on a side. On each presentation, the test line of the target configuration (the upper line for the vertical vernier, the right-hand line for oblique and horizontal verniers) was placed in one of seven positions at random. It was set either in alignment with the other line, i.e., the reference line, or misaligned by one, two, or three units to either side of the reference line. The displacement was always orthogonal to the direction of the lines. The subject's task was to identify the direction of the misalignment and to indicate his choice by setting a switch which was read by computer. The subjects were required to respond, if necessary by guessing. For the observers given feedback, an incorrect choice produced an error signal. The size of the misalignment units depended on the responses of the subject. At the end of every block of 20 trials, the 
computer was programmed to assess the number and distribution of the errors and to enlarge or reduce the misalignment unit if the number of errors indicated that the threshold lay in a different range.

\section{Training Procedure}

Each workday during training, the subjects participated in one experimental session, consisting of three 10-min runs, one for each orientation. A run produced $120-150$ responses, enough data so a preliminary threshold could be calculated and the subject informed of that day's results. The order in which the orientations were presented was varied from session to session so that after 3 days, each orientation had appeared as the first, or second, or third run of one daily session. Such a 3-day sequence constituted a practice block. The data collected from each practice block were summed and analyzed, separately by orientation, to determine the thresholds for that block. Our criterion for threshold was the offset distance at which the subject correctly identified the direction of misalignment on $75 \%$ of the presentations. We obtained the value of this distance and its standard error by fitting a probit to the psychometric function taken from the summed data. Every plotted point in our figures is based on 350450 responses, the average of three experimental sessions. The subjects were told of their progress as training proceeded. The actual length of the training period depended on the subject's schedule; every observer, however, had at least 18 days of practice (six practice blocks), amounting to more than 2,000 responses per orientation.

\section{Subjects}

Our subjects were required to have $20 / 20$ vision, with refractive corrections if needed. They were chosen from available job applicants on the basis of a 10-min test run with a vertical vernier target. Those individuals who had the best initial thresholds, usually $15 \mathrm{sec}$ of arc or less, were used as subjects. Clinical examination showed that three of our observers (M.S., S.Y., M.H.) were slightly astigmatic, the astigmatism amounting to 0.5 diopter or less in one or both eyes. Their data are included here because their best thresholds were not substantially higher for any orientation than the best thresholds obtained with the other subjects. This finding is consistent with the results of Mitchell et al. (1973), who report that astigmatic subjects do not show meridional differences greater than those found in normal eyes until the astigmatism exceeds 1.5 diopters.

\section{RESULTS}

As the curves in Figure 1 attest, every subject showed some improvement in vernier acuity with practice. Our first five subjects were given error feedback. Two additional subjects were run without error signals, and their results (Figure 2) indicate that feedback is not necessary to produce lower thresholds. Feedback was added after these two subjects had completed six practice blocks (18 days of training). It was actually disruptive for one of the subjects, although he eventually adjusted to the unexpected error signals.

The general effect of practice is shown by the curve in Figure 3. Here each point is the threshold averaged over all subjects for all three orientations and plotted as a function of the stage of training. The overall decline in threshold after 2,000-2,500 responses is about $40 \%$. There are, however, large individual variations; changes range from an in-

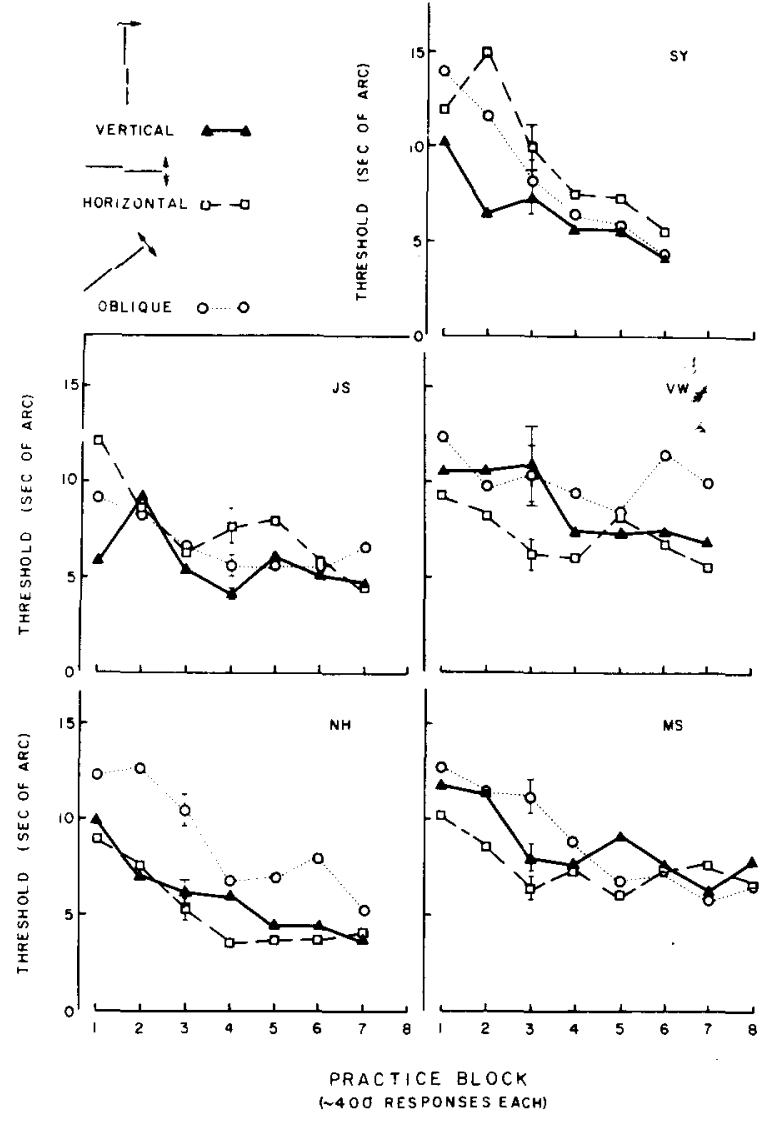

Figure 1. Vernier acuity thresholds for three target orientations for successive practice blocks in five subjects. Targets consist of two lines, each $19 \mathrm{~min}$ of arc long, presented for $220 \mathrm{msec}$. $\Delta-\Delta$ vertical vernier target, $\square-\cdots-\square$ horizontal target, $0 \cdots 0$ oblique target. Bars through symbols show representative size of \pm 1 standard error. Each practice block is made up of 350-450 responses on each orientation. Feedback given during training. All five subjects show improvement in hyperacuity with training.

significant $2 \%$ for one condition to a spectacular decrease of nearly $70 \%$ from the threshold obtained in the first practice block.

What is the influence of training on orientational differences? A two-way analysis of variance (repeated measures) of the first six practice blocks proved that both variables, practice and orientation, had statistically significant effects on the thresholds ( $p<.001$ and $p=.05$, respectively). We could conclude that orientation affects sensitivity throughout training, since the interaction between training and orientation was not significant. However, this conclusion would obscure some intriguing individual variations. The significance of the orientation effect depends on the oblique data, there being no average difference between the vertical and horizontal thresholds. The first column of numbers in Table 1 gives, for the first practice block, the ratio of the oblique vernier threshold to the average of the vertical and horizontal thresholds for each subject. 

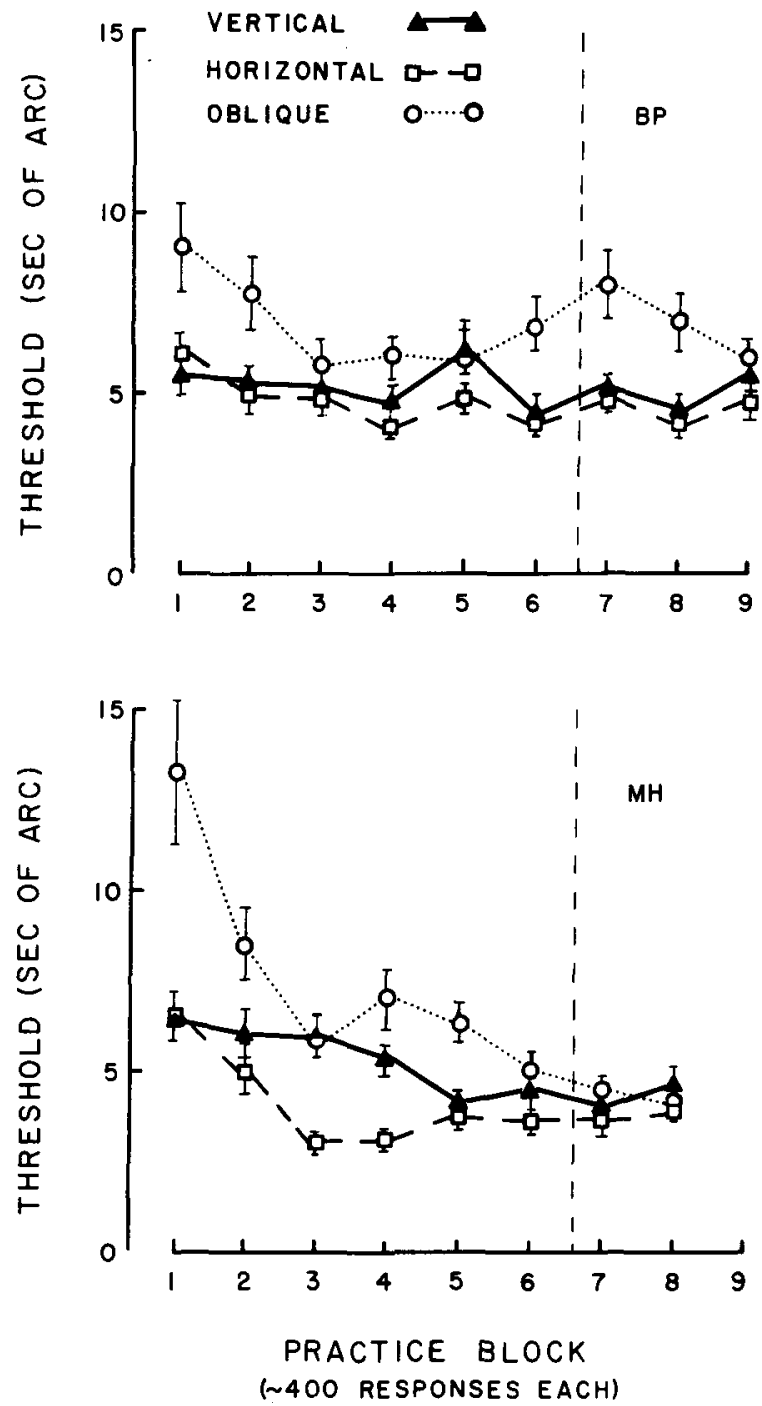

Figure 2. Vernier acuity thresholds for three target orientations as a function of the amount of practice. $\Delta-\Delta$ vertical vernier target, $\square \cdots \square$ horizontal target, $0 \cdots \cdots$ oblique target. For the first six practice blocks (350-450 responses each), no error signals were given. Dashed vertical line indicates when feedback was added.

For six of the seven subjects, the oblique threshold here is higher than the average of the other two. The magnitudes of these "oblique effects" are comparable to those observed with other acuities (Mansfield, 1974; Mitchell et al., 1966; Tyler \& Mitchell, 1977).

No simple statement could possibly be made about the ratios of the last practice block-a block which should reflect learning (second column, Table 1). For four of the subjects, the difference between the oblique targets and the vertical-horizontal targets has diminished or disappeared. The ratios for the other three subjects are the same or higher than their initial values. In fact, one of these three subjects has actually acquired an "oblique effect" during training.

The results given so far are based on specific practice procedures; they show clearly that, after 2,000-2,500 responses, the threshold for vernier acuity has declined to about $60 \%$, although orientational differences in threshold behave unpredictably from subject to subject. The two authors have noticed even greater changes in their thresholds when participating in some hyperacuity research involving many times more responses than above. Thus, the plateau that our subjects have reached after an average of $1,000-1,500$ responses probably still does not represent the optimum acuity. In view of the inconclusive nature of the results on the effect of practice on the ratio of oblique acuity to horizontalvertical acuity, we have extended the practice in a few selected subjects. Two subjects (N.H. and V.W., Figure 1) who had consistently elevated thresholds for oblique vernier acuity were given an additional 20-25 days of practice ( $1 / 2 \mathrm{~h}$ per day) with a variety of oblique targets. Their oblique vernier thresholds were virtually unchanged at the end of this period and remained higher than the values obtained with horizontal or vertical targets.

One conclusion that can be drawn from our results is that "oblique effects" should be studied with caution. Even a cursory examination of the numerous experimental papers on meridional differences shows that the magnitude of this effect varies widely among individuals possessing essentially normal vision. Our

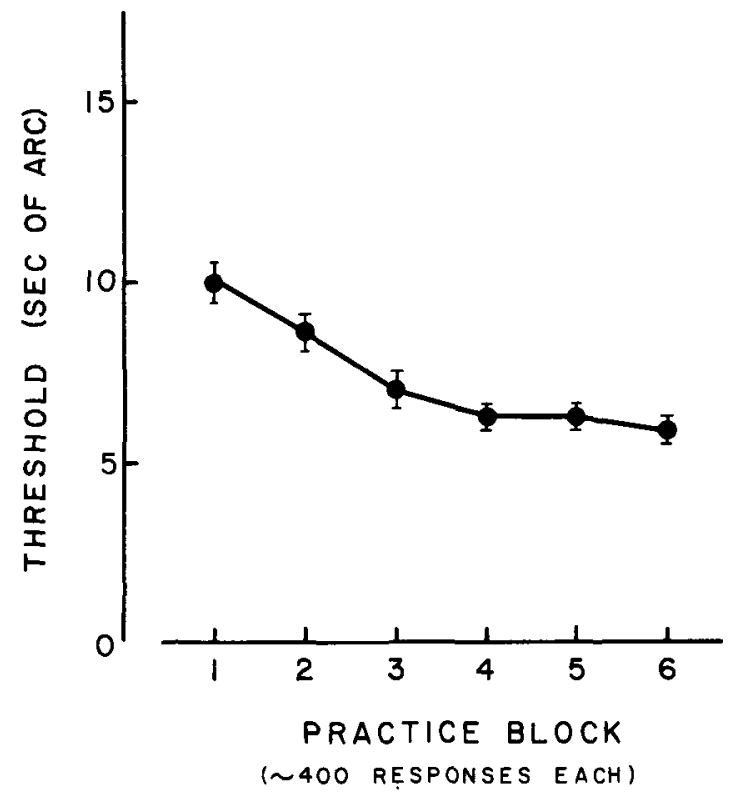

Figure 3. General learning function showing the improvement found in vernier acuity with training. Each point is the average of thresholds from all subjects for all three orientations. The overall decline is about $40 \%$. 
Table 1

Ratio of Threshold for Oblique Target to Average of Thresholds for Horizontal and Vertical Targets at Beginning of Training and at End of Training

\begin{tabular}{ccr} 
& \multicolumn{2}{c}{ Threshold Ratio } \\
\cline { 2 - 3 } Subject & \multicolumn{2}{c}{ Practice Block } \\
\hline S.Y. & 1.25 & Last \\
M.S. & 1.16 & .91 \\
V.W. & 1.24 & .92 \\
N.H. & 1.32 & 1.61 \\
J.S. & 1.02 & 1.36 \\
M.H. & 2.08 & 1.35 \\
B.P. & 1.55 & .96 \\
\hline
\end{tabular}

Note-Each block consists of 350.450 responses.

data reveal that there is also considerable individual variation in the malleability of this phenomenon. Even thresholds based on a sizable quantity of data can be misleading. A rather striking example of this problem is shown in Figure 4. These thresholds are from a subject with normal nonastigmatic eyes who participated in a pilot project for this research. The experimental conditions were very similar to those described in the Methods section except that only vertical and oblique targets were used. Also, the practice block for these data is 5 days of $\mathbf{4 0 0}$ responses each and the error is thus quite small. After three practice blocks or about 6,000 trials on each target,

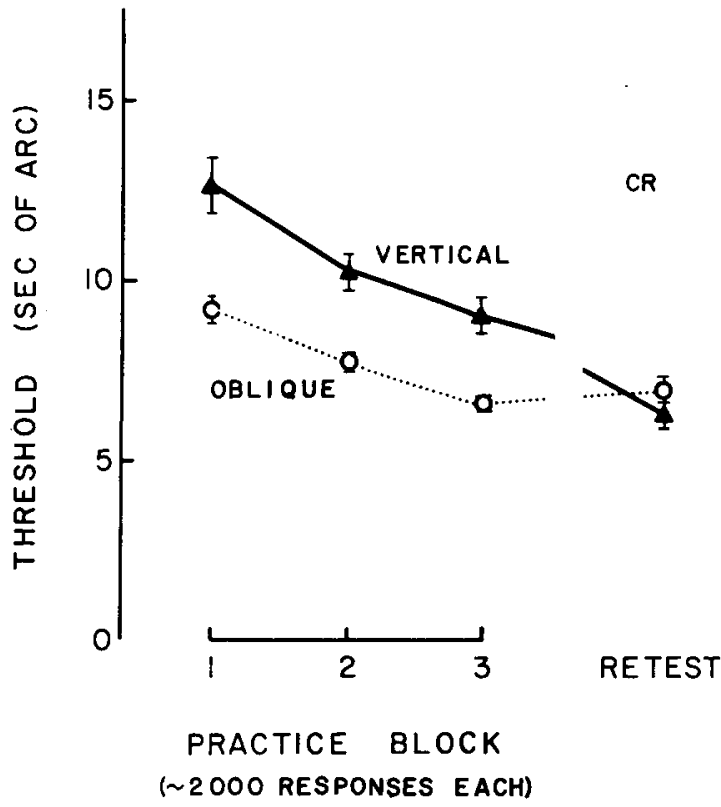

Figure 4. Vernier thresholds for subject run in pilot study using two target orientations. $\Delta-\Delta$ vertical vernier target, $0 \cdots 0$ oblique target. Practice blocks based on 5 days of training-about 2,000 trials on each orientation. Retest taken 2 months after conclusion of training, based on 1,400 responses. one could reasonably assume that this individual had better acuity for an oblique vernier target than for a vertical target. We then used this subject in a number of unrelated hyperacuity experiments which all employed vertical targets. At the end of 2 months, thresholds for these same two orientations were again measured in this subject; the "vertical effect" had disappeared.

\section{DISCUSSION}

W: believe that the substantial increase in vernier acuity shown in our results represents a genuine change in sensitivity, not just a criterional change. As with other estimates of sensitivity based on percentage correct, our thresholds can be affected by response bias (McFadden, 1970). A shift in response bias can alter a threshold when, in fact, the percentage of correct target identifications has not changed. But careful examination of the data from each subject showed that this bias effect is not responsible for the improvement found with practice. The average bias was small both at the beginning and the end of training, and the magnitude of the increase in sensitivity was in all cases much larger than the change predicted from whatever shifts in bias were found in the individual data sets.

It could be suggested that these practice effects are due to improved motor control or more accurate accommodation. Good vernier acuity, however, does not require either a steady target or sharply defined contours. In previous work, we have found that vernier thresholds are unaffected by retinal image velocities up to $2^{\circ} / \mathrm{sec}$ (Westheimer $\&$ McKee, 1975). Fine spatial localization is possible for targets with no contour displacement, the only information being a difference in the internal light distributions of the two target components (Westheimer \& McKee, 1977). Subjects are able to identify the direction of this displacement presumably by locating the relative "center of gravity" of each light distribution. We have also noted that continued experimentation has produced steady improvement in the vernier acuity of one of the authors who has little, if any, remaining accommodation.

What are the subjects learning? They tell us that they are learning to "concentrate." While some form of selective attention may be useful during training, it does not seem necessary to maintain the low values reached once training is completed. In general, welltrained subjects do not regress to the high thresholds seen in the initial stages of learning, but given much more experience, they may show still lower thresholds. Hyperacuity depends on the capacity to recognize small differences in the positions of two features whose location varies along some arbitrary spatial continuum. It seems likely that 
the improvement in hyperacuity found with practice represents a kind of "fine tuning" of whatever neural mechanism is responsible for sensing these differences.

\section{REFERENCES}

ANDRews, D. P. Perception of contour orientation in the central fovea. Part 1: Short lines. Vision Research, 1967, 7, 975-997.

BEst, F. Utber die Grenze der Erkennbarkeit von Lagenuntershieden. Albrecht v. Graefes Archiv fur Ophthalmologie, $1900,51,453-460$.

GiBson, E. J. Improvement in perceptual judgments as a function of controlled practice or training. Psychological Bulletin, 1953, 50, 401-431.

GiBson, E. J. Principles of perceptual learning and development. New York: Appleton-Century-Crofts, 1969.

GREEN, D. M., \& SwETs, J. A. Signal detection theory and psychophysics. New York: Wiley, 1966.

LEIBOWITZ, H. Some factors influencing the variability of vernier adjustments. American Journal of Psychology, 1955, 68, 226-273.

Ludvigh, E., \& McKinnon, P. The effect of orientation on the three-dot alignment test. American Joumal of Ophthalmology, $1967,64,261-265$.

MANSFIELD, R. J. W. Neural basis of orientation perception in primate vision. Science, 1974, 186, 1133-1135.
MCFADDEN, D. Three computational versions of proportion correct for use in forced choice experiments. Perception \& Psychophysics, 1970, 8, 336-342.

Mitchell, D. E., Freeman, R. D., \& Westheimer, G. Effect of orientation on the modulation sensitivity for interference fringes on the retina. Journal of the Optical Society of America, 1967, 57, 246-249.

Mrtchell, D. E., Freeman, R. D., Millodot, M., \& HAEgerstrom, G. Meridional amblyopia: Evidence for modification of the human visual system by early visual experience. Vision Research, 1973, 13, 535-558.

TanNeR, W. P., \& Rrvetre, C. L. Learning and psychophysical experiments. Journal of the Acoustical Society of , America, 1963, 35, 1896.

TYler, C. W., \& Mitchell, D. E. Orientation differences for perception of sinusoidal line stimuli. Vision Research, 1977, 17, 83-88.

Volkmann, A. W. Physiologische Untersuchungen im Gebiete der Optik. Leipzig: Breitkopf \& Härtel, 1863.

WestheImer, G., \& MCKeE, S. P. Visual acuity in the presence of retinal image motion. Journal of the Optical Society of America, 1975, 65, 847-850.

Westhelmer, G., \& MCKEE, S. P. Integration regions for visual hyperacuity. Vision Research, 1977, 17, 89-94.

WILcox, W. W. An interpretation of the relation between visual acuity and light intensity. Journal of General Psychology, $1936,15,405-435$.

(Received for publication March 4, 1978; revision accepted July 27,1978 .) 